\title{
ORIGINAL ARTICLE \\ Satisfaction with life among veterans with spinal cord injuries completing multidisciplinary rehabilitation
}

\author{
AL Fortmann ${ }^{1}$, T Rutledge ${ }^{2,3}, \mathrm{R}$ Corey McCulloch ${ }^{2,3}, \mathrm{~S} \mathrm{Shivpuri}^{1}$, AN Nisenzon ${ }^{2}$ and J Muse ${ }^{2,3}$
}

Study Design: This is a single-group, retrospective study.

Objectives: The objective of this study was to understand the factors contributing to satisfaction with life (SWL) among veterans with a spinal cord injury (SCI) completing rehabilitation.

Setting: This study was conducted at Veterans Administration Medical Center, San Diego.

Methods Between 1998 and 2010, N=118 Veterans participated in a Commission on Accreditation of Rehabilitation Facilities (CARF)-accredited rehabilitation program after a new SCl. Pre-rehabilitation measures of impairment at the organ/body level, activity limitation at the person level and participation restriction at the societal level were used to predict Satisfaction with Life Scale (SWLS) scores upon discharge.

Results: Although overall mean SWLS admission and discharge scores were not significantly different $(P>0.10)$, individual change in SWLS scores during rehabilitation was notable, ranging from a 17-point improvement to a 22-point decline across veterans (mean $\Delta=+1.18$, s.d. $=6.04$ ). Veterans who exhibited less activity limitation (higher cognitive functioning, $r=0.31, P<0.01$ ) and less participation restriction (greater social integration, $r=0.21, P<0.05$; a trend toward greater economic sufficiency, $r=0.16$, $P<0.10)$ at baseline had higher SWLS scores after rehabilitation. When these factors were entered together into a single regression model, only cognitive functioning remained statistically significant $(P<0.05)$.

Conclusion: Findings highlight potential targets for interventions, aiming to improve SWL post SCI among US veterans. In addition to directly targeting SWL with psychosocial interventions, results suggest that rehabilitation settings should continue and/or expand upon programs targeting cognitive functioning (activity limitation) and social integration (participation restriction). Nevertheless, additional research is warranted to identify the biopsychosocial factors most reliably associated with SWL and/or other aspects of quality of life. Spinal Cord (2013) 51, 482-486; doi:10.1038/sc.2012.172; published online 5 February 2013

Keywords: spinal cord injury; veterans; rehabilitation; quality of life

\section{INTRODUCTION}

Although improvements in medical management have increased the average life span post spinal cord injury (SCI), initial rehabilitation is critical, as this is when patients begin to acquire the knowledge and skills necessary to adjust to their injury and live a satisfying life. Because achieving an acceptable quality of life (QOL) is a central goal of acute SCI rehabilitation, increasing attention has been directed to assessing QOL in addition to traditional outcomes. ${ }^{1}$ Indeed, identifying predictors of $\mathrm{QOL}$ can help identify targets for indirectly enhancing QOL during rehabilitation.

Components of disability may represent particularly important influences on QOL after SCI. In 1980, the World Health Organization proposed a three-tiered conceptualization of disability named the International Classification of Impairments, Disability and Handicaps (ICIDH), ${ }^{2-3}$ which consisted of the following: impairment (that is, functional or structural abnormality of the body), disability (that is, activity limitation due to impairment) and handicap (that is, overall consequences of impairment and disability). A 1997 meta-analysis based on the ICIDH showed that measures representing handicap exhibited the strongest and most consistent inverse associations with QOL post SCI; associations of disability with QOL were also observed (yet less consistently), whereas impairment rarely predicted QOL. ${ }^{4}$ The World Health Organization subsequently revised this classification and published the International Classification of Functioning and Disability in 2002, which defines disability as a dysfunctionality at one or more of the following levels: impairment at the organ/body level, activity limitation at the person level and participation restriction at the societal level. The emphasis of 'functioning' in this model emphasizes the contribution of environmental factors- that is, disability represents an interaction between elements occurring at the personal and societal levels.

Although veterans compose $<10 \%$ of the US population based upon 2009 Census Bureau statistics, they represent a disproportionate $\sim 20 \%$ of the estimated 250000 Americans living with SCI. ${ }^{5}$ Identifying the factors most relevant to QOL in veterans with SCI could hone rehabilitative efforts for this at-need population. Research conducted to date in veterans with SCI showed older age, selfreported chronic illnesses, pain, smoking, emotional distress and lower levels of educational attainment to be linked to reduced QOL. ${ }^{6,7}$ Overall, however, research in this unique population is sparse. Further, the studies reviewed here were both cross-sectional in design and were conducted at some point after initial inpatient

${ }^{1}$ Joint Doctoral Program in Clinical Psychology, San Diego State University/University of California San Diego, San Diego, CA, USA; ${ }^{2}$ VA San Diego Healthcare System, San Diego, CA, USA and ${ }^{3}$ Department of Psychiatry, University of California San Diego, San Diego, CA, USA

Correspondence: Dr AL Fortmann, Joint Doctoral Program in Clinical Psychology, San Diego State University/University of California, San Diego, 9245 Sky Park Court no. 105, San Diego, CA 92123, USA.

Email: abrewer@projects.sdsu.edu

Received 9 February 2012; revised 9 November 2012; accepted 14 November 2012; published online 5 February 2013 
rehabilitation-the period of time that has been highlighted as especially predictive of long-term SCI adjustment. ${ }^{8}$ More broadly, no study, to our knowledge, has conducted a rigorous comparison of the differential utility of impairment, activity limitation and participation restriction in predicting QOL in a single sample of individuals (nevertheless veterans) with SCI. It is important to acknowledge that QOL is a broad factor including both objective and subjective components. A recent meta-analysis ${ }^{9}$ investigating QOL in SCI identified 9 distinct (2 objective, 7 subjective) QOL measures used in published research with SCI populations. Among these measures is the Satisfaction with Life Scale (SWLS), a brief assessment of subjective QOL.

The current, retrospective study begins to address these gaps in the literature by investigating predictors of post-rehabilitation, subjective QOL (heretofore, satisfaction with life (SWL)) among a sample of veterans participating in a multidisciplinary SCI rehabilitation program. Our specific aims were to 1) describe change in SWL from preto post-rehabilitation; 2) compare indicators of impairment, activity limitation and participation restriction in prospectively predicting SWL post rehabilitation; and 3) develop a statistical model for predicting SWL that can potentially improve rehabilitation emphases. Hypotheses were informed by the 1997 meta-analysis, ${ }^{4}$ which based conclusions about predictors of QOL on findings from several independent studies (each evaluating one or more components of the 1980 ICIDH). Using the revised 2002 International Classification of Functioning and Disability, we predicted that participation restriction and activity limitation would exhibit the strongest and second-strongest inverse associations with SWL, respectively; we predicted that impairment would not be a significant predictor of SWL.

\section{MATERIALS AND METHODS}

\section{Procedures}

Of the 121 veterans admitted for acute SCI rehabilitation at the VA San Diego Healthcare System during 1998 and 2010, N=118 were selected to partake in Commission on Accreditation of Rehabilitation Facilities (CARF)-accredited inpatient rehabilitation (based on perceived ability to benefit from this highly structured program) and were included in the present analyses. Rehabilitation was individualized, lasted up to several months and involved several interdisciplinary SCI-specific providers. Study measures were routine clinical assessments performed as part of each patient's admission and discharge procedures. The University of California, San Diego Institutional Review Board and the VA San Diego Healthcare System granted approval for the study.

\section{Measures}

Satisfaction with Life. The SWLS, ${ }^{10}$ a measure of subjective QOL, assesses individuals' satisfaction with their present status as compared with a standard that each individual sets for him/herself (that is, the standard is not externally imposed). Possible scores ranged from 5 to 35 , with higher scores indicating greater SWL. In the present study, SWLS was also examined categorically; scores $\geqslant 20$ indicated 'average' or better SWL, according to scoring guidelines. ${ }^{11}$ The SWLS has exhibited good internal consistency $(\alpha=0.80-$ $0.89)$, test-retest reliability $(r>0.80)^{10}$ and sensitivity to change among rehabilitation patients. ${ }^{12}$

Components of functioning and disability. Potential predictors of SWL were assessed upon admission to the rehabilitation program (that is, baseline) and classified according to the three International Classification of Functioning and Disability categories: impairment, activity limitation and participation restriction.

Impairment. Impairment was represented by injury level (tetraplegia $=1$, paraplegia $=0)$ and completeness of injury, according to the American Spinal Injury Association Impairment Scale $(\mathrm{A} /$ complete $=1$ to $\mathrm{E} /$ normal $=5)$.
Activity limitation. The Functional Independence Measure (FIM) ${ }^{13}$ is a wellvalidated assessment of physical and cognitive disability, ${ }^{14}$ which is widely utilized in rehabilitation settings. A total of 18 items were clinician rated on a 1-7 scale and summed to form motor (11 items), elimination ( 2 items) and cognition (5 items) subscales. Higher scores reflected less activity limitation (greater activity).

Participation restriction. Veterans completed the Craig Handicap Assessment and Reporting Technique (CHART), ${ }^{15}$ a well-validated, ${ }^{16}$ 27-item measure of the degree to which impairments and/or activity limitations result in restriction in the following domains: 1) physical independence; 2) mobility; 3) occupation; 4) social integration; and 5) economic self-sufficiency. Each CHART dimension is scored $0-100$, with higher scores indicating less participation restriction (greater participation).

\section{Statistical analyses}

Data were derived from a database that was created by the San Diego VA Medical Center in 1998 following a national conference held to prepare VA SCI centers for the CARF accreditation process. To examine Aim 1, repeated measures ANOVA was conducted to examine group-level change from admission to discharge in mean SWLS scores; frequencies were also obtained to describe individual-level change in SWL. To address Aim 2, bivariate correlation analyses examined associations of potential predictors (assessed at baseline) with SWLS discharge scores. Next, baseline indicators that exhibited at least a trend $(P<0.10)$ toward statistical significance in these (exploratory) analyses were entered jointly into a multiple linear regression model to examine the relative utility of each in predicting SWLS discharge scores. Finally, for Aim 3, a regression model containing only the significant predictor(s) from the latter analysis was used to determine beta weights for a prediction algorithm. All regression analyses controlled for baseline SWLS scores and patient age; statistical significance was declared as $P<0.05$. All analyses were conducted using the SPSS software, version 17.0 (SPSS Inc., Chicago, IL, USA).

\section{RESULTS}

\section{Descriptive statistics}

The age of patients ranged from 18 to 80 years $(\mathrm{M}=43.16 \pm 17.54)$; the majority were Caucasian (65\%), male (97\%) and entered inpatient rehabilitation $\leqslant 60$ days post injury (80\%) (Table 1). Bivariate associations among baseline predictors and SWLS discharge scores are presented in Table 2.

\section{Pre- to post-rehabilitation change in SWLS scores}

At the aggregate level, there was no statistically significant difference between mean SWLS scores at admission and discharge (22.70 \pm 7.28 versus $21.52 \pm 7.78, P>0.10)$; both means were just above the 'average' life satisfaction cutoff (SWLS $=20$ ). At the individual level, $14 \%$ of participants evinced a decrease from 'at or above average' $(S W L S \geqslant 20)$ to 'below average' (SWLS $<20$ ), whereas $17 \%$ showed an increase in SWLS from 'below average' to 'at or above average' from admission to discharge; $69 \%$ of participants remained in the same category over this time period. The range of individual change in SWLS scores spanned from -22 to +17 across patients (mean individual $\Delta=+1.18$, s.d. $=6.04$; Figure 1).

\section{Predictors of SWLS scores at discharge}

Statistically (or marginally) significant bivariate associations were observed between higher SWLS scores at discharge and higher FIM cognition scores $(r=0.31, P<0.01)$, and higher CHART social integration $(r=0.21, \quad P<0.05)$ and economic self-sufficiency $(r=0.16, P<0.10)$ scores at baseline (Table 2). When these three baseline variables were entered together into a regression model predicting discharge SWLS scores, only FIM cognition $(P<0.05)$ 
Table 1 Descriptive statistics for all demographic and study variables $(N=118)^{\mathrm{a}}$

\begin{tabular}{|c|c|}
\hline Variable & $\%$ \\
\hline \multicolumn{2}{|l|}{ Sample characteristics } \\
\hline Male & 97.46 \\
\hline Caucasian & 65.25 \\
\hline Employed for wages & 43.22 \\
\hline Diagnosed substance use or other psychiatric disorder & 20.31 \\
\hline$\leqslant 60$ days from injury to admission & 79.70 \\
\hline \multicolumn{2}{|l|}{ Cause of injury } \\
\hline MVA & 36.44 \\
\hline Violence & 3.39 \\
\hline Sports & 6.78 \\
\hline Falls & 17.80 \\
\hline Circulatory & 5.93 \\
\hline Spinal cord compression & 15.25 \\
\hline Other & 14.41 \\
\hline Sustained injury in combat & 2.50 \\
\hline Traumatic brain injury ${ }^{\mathrm{b}}$ & 5.10 \\
\hline \multicolumn{2}{|l|}{ Impairment } \\
\hline Tetraplegic & 54.71 \\
\hline ASIA A & 37.93 \\
\hline Activity limitation (FIM) & Mean (s.d.) \\
\hline Cognition ${ }^{c}$ & $31.39(3.87)$ \\
\hline Motord & $24.53(13.50)$ \\
\hline Elimination ${ }^{\mathrm{e}}$ & 4.15 (3.78) \\
\hline \multicolumn{2}{|l|}{ Participation restriction $(C H A R T)^{f}$} \\
\hline Physical independence & $98.55(7.20)$ \\
\hline Mobility & $94.29(16.89)$ \\
\hline Occupation & $85.42(28.96)$ \\
\hline Social integration & $93.41(16.38)$ \\
\hline Economic self-sufficiency & $87.17(23.07)$ \\
\hline \multicolumn{2}{|l|}{ SWLS } \\
\hline Admission & $22.70(7.28)$ \\
\hline Discharge & $21.52(7.78)$ \\
\hline \multicolumn{2}{|c|}{ 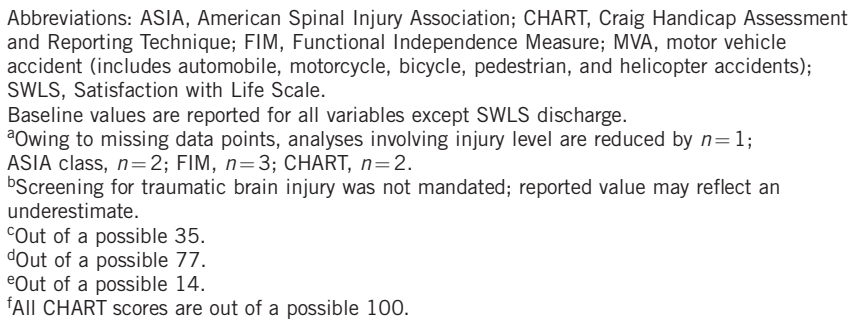 } \\
\hline
\end{tabular}

remained a statistically significant predictor after controlling for age and baseline SWLS scores. (Additional control variables in the form of etiology of injury, time since injury and psychiatric comorbidity were included in follow-up analyses. Adjustment for these variables had no appreciable effect on the results, and therefore the simpler final model was maintained).

\section{Statistical model for predicting SWLS scores at discharge}

The following weighted formula was extracted from final regression analyses to statistically predict discharge SWLS scores: [Discharge
SWLS $=1.18+0.60 \quad$ (Admission SWLS) -0.03 (age) $+0.31 \quad(\mathrm{FIM}$ cognition)]. After accounting for variance explained by covariates (age and baseline SWLS; 46.2\%, $P<0.001$ ), FIM cognition scores explained an additional $2.1 \%(P=0.02)$ of the variance in SWLS scores at discharge (Total adjusted $R^{2}=0.48$ ). Exploratory analyses confirmed the stability of the FIM cognition predictor across meaningful subgroups (that is, age median split; tetraplegia versus paraplegia); separate interaction terms representing 'age by FIM cognition' and 'injury level by FIM cognition' were not statistically significant when added to the model (both $P>0.10$ ).

\section{DISCUSSION}

This study is the first, to our knowledge, to investigate the relative utility of impairment, activity limitation and participation restriction in predicting SWL among veterans completing acute rehabilitation for SCI. We observed marked individual variability in the magnitude and direction of SWL changes from pre- to post-rehabilitation, but average SWL changes were small and not statistically significant. Consistent with previous literature ${ }^{4}$ and a priori predictions, exploratory correlation analyses showed that measures of activity limitation at the person level (that is, cognitive impairment) and participation restriction at the societal level (that is, social integration) were the strongest predictors of SWL changes; the association between economic self-sufficiency (another indicator of participation restriction) and SWL approached statistical significance. When these indicators were entered jointly in a regression model, activity limitation (versus participation restriction, as originally hypothesized and as observed in previous studies ${ }^{4}$ ) emerged as the most robust predictor of SWL.

Multidisciplinary rehabilitation strives to help veterans effectively manage their disability while learning new strategies and assistance technology to enhance independence. Maximizing function and SWL are particularly important rehabilitation goals. Studies improving upon the existing understanding of SWL post SCI have the potential to improve existing rehabilitation programs by guiding intervention efforts toward the factors most reliably associated with favorable outcomes.

The link between cognitive impairment (activity limitation) and SWL in the present study supports the growing importance attributed to cognitive functioning in recent years. Cognitive screening is a standard component of acute SCI rehabilitation at VA Medical Centers; veterans are referred for cognitive rehabilitation to retrain skills and/or develop compensatory strategies to reduce functional impairment as indicated. However, because individuals with higherlevel SCIs are unable to complete the manual portions of many standard neuropsychological tests, cognitive screening approaches must be flexible. Further, many cognitive rehabilitation tools (for example, electronic mobile devices) require modifications. Consistent with findings pertaining to social integration (participation restriction), veterans are offered assistance with building new and/or mobilizing existing social support resources during or after rehabilitation. Support groups and peer-mentor programs for individuals with SCI represent example methods by which veterans can integrate with others coping with similar adjustment difficulties. Given research showing that early adjustment post SCI often predicts long-term adjustment ${ }^{8}$, rehabilitation programs should also maintain or expand upon programs that more directly target various aspects of emotional well-being, including SWL. A systematic review supported the use of specialized CBT protocols in persons with SCI experiencing depression, anxiety and adjustment and coping difficulties. ${ }^{17}$ In addition to increasing access, time efficiency and 
Table 2 Correlations among study variables $(N=118)^{\mathrm{a}}$

\begin{tabular}{|c|c|c|c|c|c|c|c|c|c|c|c|}
\hline & 1 & 2 & 3 & 4 & 5 & 6 & 7 & 8 & 9 & 10 & 11 \\
\hline Injury level & -0.04 & 1.00 & & & & & & & & & \\
\hline CHART physical independence & 0.04 & 0.15 & $-0.16^{*}$ & 1.00 & & & & & & & \\
\hline CHART mobility & 0.09 & -0.07 & $-0.23^{* *}$ & $0.53^{* * *}$ & 1.00 & & & & & & \\
\hline CHART occupation & 0.11 & -0.06 & $-0.27^{* * *}$ & $0.34^{* * *}$ & $0.61^{* * *}$ & 1.00 & & & & & \\
\hline FIM cognition & $0.31^{* * *}$ & 0.10 & 0.08 & -0.12 & 0.12 & 0.09 & $0.33^{* * *}$ & 0.12 & 1.00 & & \\
\hline FIM motor & -0.03 & $-0.29 * * *$ & $0.22^{* *}$ & -0.06 & -0.05 & 0.01 & -0.05 & 0.06 & $0.26^{* * *}$ & 1.00 & \\
\hline FIM elimination & 0.12 & 0.06 & $0.43^{* * *}$ & $-0.30^{* * *}$ & $-0.17^{*}$ & -0.03 & -0.06 & 0.07 & $0.25^{* * *}$ & $0.69^{* * *}$ & 1.00 \\
\hline
\end{tabular}

Abbreviations: CHART, Craig Handicap Assessment and Reporting Technique; FIM, Functional Independence Measure; SWLS, Satisfaction With Life Scale.

${ }^{*} P<0.10,{ }^{* *} P<0.05$. ${ }^{* * *} P<0.01$.

Higher CHART and FIM scores reflect less handicap and disability, respectively.

aOwing to missing data points, analyses involving injury level are reduced by $n=1$; ASIA class, $n=2$; FIM, $n=3$; CHART, $n=2$.

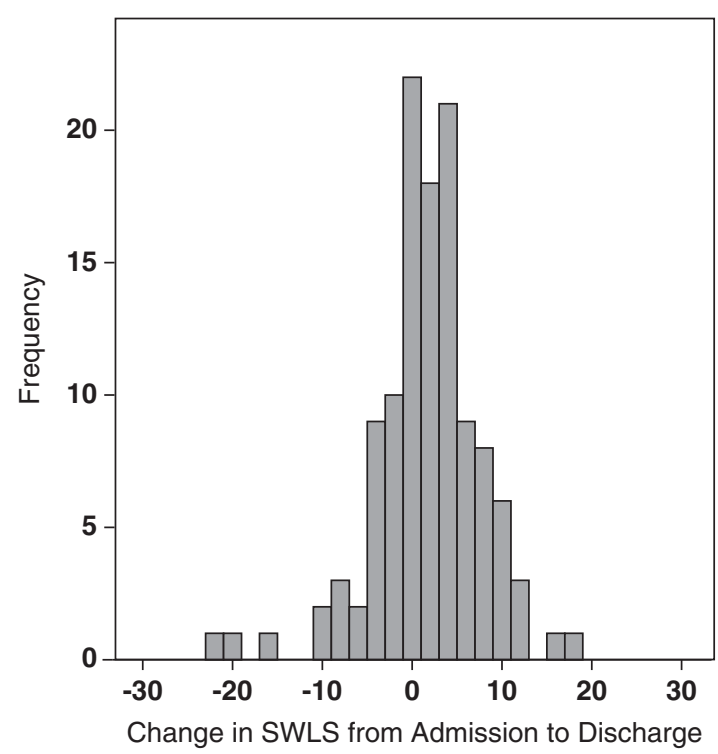

Figure 1 Individual change in SWLS scores from admission to discharge from rehabilitation. Positive and negative scores indicate improvement and decline, respectively; scores of 0 indicate no change in SWLS scores over this time period.

cost-effectiveness, ${ }^{18}$ telecounseling interventions may be an especially valuable medium for the provision of continued services to outpatients who have limited mobility (for example, SCI patients post inpatient rehabilitation). A recent meta-analysis found that telephone-administered psychological interventions improved QOL, coping skills, community integration and depression in individuals with SCI and other acquired (permanent) physical disabilities. ${ }^{19}$

\section{Study limitations}

Several caveats should be considered in the interpretation of these results. First, findings pertain to a particular definition/measure of subjective QOL, SWL, and cannot be generalized to other QOL conceptualizations. Second, the limited change in SWL at the group level was unexpected and suggests a variety of potentially overlapping interpretations. To the extent that our results reflect a limitation of the SWLS, measures of different aspects of QOL could yield different results. Furthermore, the individual-level changes in SWLS scores are difficult to interpret as increases (for example) in SWL, could be attributable to actual status improvement over time or to a lowering of standards by which individuals use to judge such progress. ${ }^{20}$ Third, because analyses were limited to veterans selected to partake in CARF-accredited, inpatient rehabilitation, generalizability to other SCI veteran populations may be compromised. Next, this study examined SWL over the course of acute, inpatient rehabilitation. Although early post-injury adjustment is critical, ${ }^{8}$ SWL and other aspects of QOL remain important considerations as one adjusts to life outside of the hospital. As such, future studies should examine the trajectory of SWL, and predictors thereof (especially environmental factors such as physical, attitudinal and policy barriers ${ }^{3,21}$ ), beyond inpatient rehabilitation. Limitations of predictor variables should also be noted. The reliability and validity of FIM scores, for example, are influenced by clinician training and education; FIM subscale reliability may also vary by individual subscale length. Because FIM and CHART scales are not SCI specific, they can be prone to ceiling/ floor effects that constrain scale variability and limit the ability to detect associations. Nevertheless, prior studies of patients with SCI support their use in SCI and other rehabilitation populations. ${ }^{21,22}$ Finally, although we attempted to include a relatively comprehensive set of predictors based on prior SCI research, it is possible that the omission of additional factors caused us to miss important correlates of SWL changes. From our perspective, however, limitations in the predictor variables had only modest potential for influence, given that there was little SWLS change to predict.

\section{CONCLUSIONS}

Veterans completing acute, inpatient SCI rehabilitation averaged only modest pre- to post-program changes in SWL. We observed significant associations of activity limitation at the person level (that is, cognitive impairment) and participation restriction at the societal level (that is, social integration, economic self-sufficiency) with SWL post rehabilitation; however, only the former was statistically significant when considered jointly. Overall, this study highlights potential targets for interventions aiming to improve SWL post SCI among US veterans. Nevertheless, these findings also suggest the need for additional research to identify the aspects of QOL that are most amenable to change during rehabilitation, and to explore factors that contribute most reliably to these changes. 


\section{DATA ARCHIVING}

There were no data to deposit.

\section{CONFLICT OF INTEREST}

The authors declare no conflict of interest.

1 Hill MR, Noonan VK, Sakakibara BM, Miller WC. Quality of life instruments and definitions in individuals with spinal cord injury: a systematic review. Spinal Cord 2010; 48: 438-450.

2 World Health Organization. International Classification of Impairments, Disabilities and Handicaps: A Manual of Classification Relating to the Consequences of Disease. World Health Organization: Geneva, 1980.

3 World Health Organization. International Classification of Functioning, Disability, and Health. World Health Organization: Geneva, 2002.

4 Dijkers M. Quality of life after spinal cord injury: a meta analysis of the effects of disablement components. Spinal Cord 1997; 35: 829-840.

5 US Department of Veterans Affairs, Office of Public Affairs. Fact sheet: VA and Spinal Cord Injury, 2009. Department of Veterans Affairs: Washington, DC. Accessed Feb 2 2012, available at http://controlbionics.com/Fact\%20Sheet\%20SCl\%202009.pdf.

6 Smith BM, LaVela SL, Weaver FM. Health-related quality of life for Veterans with spina cord injury. Spinal Cord 2008; 46: 507-512.

7 Martz E, Livneh H, Priebe M, Wuermser LA, Ottomanelli L. Predictors of psychosocial adaptation among people with spinal cord injury or disorder. Arch Phys Med Rehabil 2005; 86: 1182-1192.

8 Pollard C, Kennedy P. A longitudinal analysis of emotional impact, coping strategies and post-traumatic psychological growth following spinal cord injury: a 10-year review. Brit J Health Psych 2007; 12: 347-362.

9 Ravenek KE, Ravenek MJ, Hitzig SL, Wolfe DL. Assessing quality of life in relation to physical activity participation in persons with spinal cord injury: a systematic review. Disabil Health J 2012; 5: 213-223.

10 Diener E, Emmons RA, Larsen RJ, Griffin S. The Satisfaction With Life Scale. J Pers Assess 1985; 49: 71-75.
11 Diener E. Understanding Scores on the Satisfaction with Life Scale. Accessed July 1 2012, available at http://internal.psychology.illinois.edu/ ediener/Documents/ Understanding\%20SWLS\%20Scores.pdf).

12 Ruff RL, Adamson VW, Ruff SS, Wang X. Directed rehabilitation reduces pain and depression while increasing independence and satisfaction with life for patients with paraplegia due to epidural metastatic spinal cord compression. J Rehabil Res Dev 2007; 44: 1-10.

13 Uniform Data Set For Medical Rehabilitation. Guide for the Use of the Uniform Data Set for Medical Rehabilitation, Version 5.0. State University of New York at Buffalo Research Foundation: Buffalo, NY, 1996.

14 Cohen ME, Marino RJ. The tools of disability outcomes research functional status measures. Arch Phys Med Rehabil 2000; 81(Suppl 2): S21-S29.

15 Whiteneck GG, Charlifue SW, Gerhart KA, Overholser JD, Richardson GN. Quantifying handicap: a new measure of long-term rehabilitation outcomes. Arch Phys Med Rehabil 1992; 73: 519-526.

16 Mellick D. The Craig Handicap Assessment and Reporting Technique. The Center for Outcome Measurement in Brain Injury. 2000. Accessed Oct 4 2012, available at http://www.tbims.org/combi/chart.

17 Mehta S, Orenczuk S, Hansen KT, Aubut JA, Hitzig SL, Legassic M et al. An evidence-based review of the effectiveness of cognitive behavioral therapy for psychosocial issues post-spinal cord injury. Rehabil Psychol 2011; 56: $15-25$.

18 Bendixen RM, Levy CE, Olive ES, Kobb RF, Mann WC. Cost effectiveness of a telerehabilitation program to support chronically ill and disabled elders in their homes. Telemed J E-Health 2009; 15: 31-38.

19 Dorstyn DS, Mathias JL, Denson LA. Psychosocial outcomes of telephone-based counseling for adults with an acquired physical disability: a meta-analysis. Rehabil Psychol 2011; 56: 1-14.

20 Dijkers MP. Correlates of life satisfaction among persons with spinal cord injury. Arch Phys Med Rehabil 1999; 80: 867-876.

21 Whiteneck G, Meade MA, Dijkers M, Tate DG, Bushnik T, Forchheimer MB. Environmental factors and their role in participation and life satisfaction after spinal cord injury. Arch Phys Med Rehabil 2004; 85: 1793-1803.

22 Anderson K, Aito S, Atkins M, Biering-Sorensen F, Charlifue S, Curt A et al. Functional recovery measures for spinal cord injury: an evidence-based review for clinical practice and research. J Spinal Cord Med 2008; 31: 133-144. 